\title{
COVID 19 and Heart
}

\author{
Abdullah Al Shafi Majumder \\ Professor of Cardiology, Ex-Director, National Institute of Cardiovascular Diseases, Dhaka
}

(Cardiovasc.j. 2020; 13(1): 3-4)

Like any other severe illness due to respiratory virus, SARS-CoV-2 in severe form is associated with myocardial injury with features of myocarditis with ECG changes and increased level of troponin. But it involves myocardium at far more rate (24-33\%) than influenza (around 1\%). It is understood when we consider the pathophysiology of the way virus acts in the body. It utilizes Angiotensin Converting Enzyme 2 (ACE2) receptors to enter the cells. These receptors are abundant in the lungs and much expressed in the myocardium, endothelium. kidneys and GI tract. Following a few days of infection, it enters myocytes causing myocardial injury. This direct effect of the virus leads to focal myocardial damage and scar formation. Thus, in addition to immediate cause of arrhythmia, it remains as nidus of future arrhythmia. In the later stage of the diseases, when there is immune response with cytokine release, there is suppression of myocardium with onset of acute left ventricular failure. This process of myocardial suppression is transient with no residual damage to the myocardium in most cases.

Persons with cardiovascular diseases are more prone to SARS-CoV-2 and when infected run a worse course. Any comorbid condition is associated with increased fatality rate and with cardiovascular comorbidity it is reported to be as high as $10.5 \%$. With this understanding our advice to a patient with cardiovascular diseases is number one- take extra precautions to avoid to get infected and number two is to continue the cardiac drugs stringently. Properly managed patients appear to go better way than the persons who are ill managed.

Anti-hypertensive /anti-failure drugs including angiotensin converting enzyme inhibitors (ACEI) $\&$ angiotensin receptor blockers (ARB), betablockers and diuretics are to continued. Initially there was fear that as the virus enters the cell by binding with angiotensin converting enzyme 2 (ACE2) and as there is upregulation of these receptors on the use ACEI/ARB, these may facilitate the cellular entry of the virus. No doubt, it was a theoretical possibility but several observers found that clinically these do no harm. After a series of discussion all the experts extend no harm chit. Some claim the RAAS inhibitors including ARNI may provide safety effects which is not widely accepted. Drugs prescribed for ischemic heart diseases - anti-platelets and statins need to be continued. Prescription of anticoagulants during illness necessitates adjustment of dual antiplatelet therapy. There is a view by some authors that statins may have additional benefit which await further investigation.

Cardiac manifestations are myocardial injury as evident by rise of troponin I/T, myocarditis, acute myocardial infarction - both type 1 and type 2, stress cardiomyopathy (Taku-Tsubo cardiomyopathy), arrhythmias, acute heart failure, pericarditis \& pericardial effusion, hypotension \& shock. Those patients who have prior cardiovascular diseases show more cardiac complications. Some of the manifestations are secondary to hypoxia due to lung causes such as type 2 myocardial infarction. Identifying the cardiac manifestation appropriately is the key to proper cardiological advice. 12 lead resting ECG provides diagnostic information along with measurement of cardiac biomarkers. These need periodic assessment during whole period of illness. Echocardiogram provides decisive information in this situation but the test should be advised only when treatment is going to be impacted by the findings. As per recommendation from the concerned society, echo should be point of focus with all the protective measures to the health care providers. Examination should be brief though collecting all the information for the patient, echo findings should be recorded for interpretation after finishing physical presence of the patients.

One major issue is how to treat ST elevated acute myocardial infarction. It is stated that recommendation for standard care that is primary PCI is not altered but other considerations to be 
kept in mind. In this pandemic era, golden time period of 90 or 120 minutes for primary PCI is delayed in many cases. Triage of the patients will take time that includes test for SARS-CoV-2 and the cath lab and the personnel taking safety measures will take more than usual time. Suggestion to have a dedicated cath lab for the COVID 19 patients is hard to follow and almost impossible in third world countries. Considering this, fibrinolytic based therapy is highlighted, which was suggested from experience from Wuhan, and widely accepted by the many centres.

Due to hypercoagulable state there are thrombotic complications manifested in different organs including cardiovascular system. Type 1 myocardial infarction in COVID 19 may be due to thrombotic occlusion; increased thrombus burden has been reported in acute STEMI patients and there are reports of increased incidence of stent thrombosis. Thus, anticoagulants generally recommended for the patients are useful for cardiac involvement.

As we passed more than 7 months from the outbreak of the disease, there are now reports to show the aftermath of the cardiac (also pulmonary) involvement. Certain percentage of the patients have reduced ejection fraction and there is fibrous scar (potential source of arrhythmias) of myocardial. We expect more data on the residual cardiac effects in the ensuing months to have a definite idea. In the mean time these findings send a message of warning to the world of sports. Every athlete/ sportsman who recovered from COVID 19 need to be thoroughly undergone cardiac assessment before they go to restart training. They need close observation during training and during game for any adverse event.

COVID 19 comes out as a cardiac disease as importantly as pulmonary diseases. Prognosis of the patients is determined significantly by the cardiac involvement. Reported delayed effects on heart is a serious dimension of the course of the disease. In the coming months we will have more experiences and more information about the diseases that will make us more prepared to deal with the disease.

\section{Conflict of Interest - None.}

\section{References:}

1. Clerkin KJ, Fried JA, Raikhelkar J, Sayer G, Griffin JM, Masoumi A, et al. COVID-19 and Cardiovascular Disease. Circulation 2020; 141(20): 1648-1655.
2. Zheng Y, Ma Y, Zhang J, et al. COVID-19 and the cardiovascular system. Nat Rev Cardiol 2020; 17: 259260. doi.org/10.1038/s41569-020-0360-5

3. Xiong TY, Redwood S, Prendergast B, Chen M. Coronaviruses and the cardiovascular system: acute and long-term implications. Eur Heart J 2020; 41:17981800. doi:10.1093/eurheartj/ehaa231

4. Fried JA, Ramasubbu K, Bhatt R, Topkara VK, Clerkin KJ, Horn E, et al. The Variety of Cardiovascular Presentations of COVID-19. Circulation 2020; 141(23): 1930-1936.

5. Driggin E, Madhavan MV, Bikdeli B, et al. Cardiovascular Considerations for Patients, Health Care Workers, and Health Systems During the COVID19 Pandemic. J Am Coll Cardiol 2020; 75(18): 23522371.

6. Prabhakaran D, Perel P, Roy A, Singh K, Raspail L, et al. Management of Cardiovascular Disease Patients With Confirmed or Suspected COVID-19 in Limited Resource Settings. Global Heart 2020; 15(1): 44. DOI: https://doi.org/10.5334/gh.823

7. Mehra MR, Desai SS, Kuy SR, Henry TD, Patel AN: Cardiovascular Disease, Drug Therapy and Mortality in Covid-19. N Engl J Med 2020. DOI: 10.1056/ NEJMoa2007621

8. The European Society for Cardiology. ESC Guidance for the Diagnosis and Management of CV Disease during the COVID-19 Pandemic. https:// www.escardio.org/Education/COVID-19-andCardiology/ESCCOVID-19-Guidance. (Last update: 10 June 2020)

9. Abajo FJ, Martín SR, Lerma V, et al. on behalf of the MED-ACE2-COVID19 study group. Use of reninangiotensin-aldosterone system inhibitors and risk of COVID-19 requiring admission to hospital: a casepopulation study. Lancet 2020; 395: 1705-1714.

10. Sud K, Vogel B, Bohra C, Garg V, Talebi S, Lerakis S, et al. Echocardiographic Findings in COVID-19 Patients with Significant Myocardial Injury. J Am Soc Echocardiogr 2020. doi.org/10.1016/j.echo.2020.05.030

11. Inciardi RM, Adamo M, Lupi L, et al. Characteristics and outcomes of patients hospitalized for COVID-19 and cardiac disease in Northern Italy. Eur Heart $J$ 2020; 41: 1821-1829. doi:10.1093/eurheartj/ehaa388

12. Guidance on Cardiac Implications of COVID-19 (ACC, 2020). Medscape - Mar 25, 2020

13. Gao C, Cai Y, Zhang K, et al. Association of hypertension and antihypertensive treatment with COVID-19 mortality: a retrospective observational study. Eur Heart J 2020; 41: 2058-2066. doi:10.1093/eurheartj/ehaa433

14. Mehta N, Kalra A, NowackI AS, et al. Association of Use of Angiotensin-Converting Enzyme Inhibitors and Angiotensin II Receptor Blockers with Testing Positive for Corona virus Disease 2019 (COVID-19). JAMA Cardiol 2020; doi:10.1001/jamacardio.2020.1855

15. Aggarwal G, Cheruiyot I, Aggarwal S, et al. Association of Cardiovascular Disease with Coronavirus Disease 2019 (COVID-19) Severity: A Meta-Analysis. Curr Probl Cardiol 2020. doi: https://doi.org/10.1016/j.cpcardiol. 2020.100617 